\title{
Framing abuse: explaining the incidence, perpetuation, and intervention in elder abuse
}

\author{
Yuliya Mysyuk, Rudi G. J. Westendorp and Jolanda Lindenberg \\ Leyden Academy on Vitality and Ageing, Poortgebouw LUMC, Rijnsburgerweg 10, 2333 AA Leiden, the Netherlands
}

ABSTRACT

Background: The role of individual characteristics in incidences of elder abuse has long been highest on research and policy agendas. Now, it is timely to discuss factors that go beyond victim and perpetrator. Environmental factors also play an important role in elder abuse. In this paper, we address the framing of elder abuse as a social and health problem. Attention is paid to the factors that influence societal context and the healthcare system, its organization, structure, and principles.

Methods: Focus groups and in-depth semi-structured interviews were held with different professionals and older people themselves. Qualitative analysis of focus groups and interviews transcripts was performed to analyze how different professional groups and older persons themselves view elder abuse, to determine opinions and attitudes toward elder abuse and the necessary actions that should be taken to prevent or intervene in the problem.

Results: Two main explanatory frameworks emerged in the discourse of older persons and care professionals: social arrangements and healthcare system. The themes within the social arrangements included social taboo, social control and responsibility, and institutional cultures. The fragmentation of care and changes in the financing of healthcare were two aspects distinguished within the framework of the healthcare system.

Conclusion: Two explanatory frameworks showed elder abuse as both a social and health problem. The environmental factors through social arrangements and healthcare system have an influence on framing of abuse. The different ways of framing abuse impact the understanding of abuse, ways of intervention, and prevention measures.

Key words: elder abuse, qualitative research, framing, environmental factors, healthcare, neoliberalism

\section{Introduction}

Previously scientific research on elder abuse focused primarily on individual characteristics of victims and perpetrators. Considering the in-depth research that has already been done on perpetratorvictim characteristics (see, for instance, Lachs and Pillemer, 2004; Hörl, 2010), it is timely to pay more attention to the relationship and interaction between victim and perpetrator, to environmental factors, which can in fact play a bigger role than previously assumed and studied in the context of elder abuse.

Factors in the environment can be viewed as mirroring the social institutions that are

Correspondence should be addressed to: Yuliya Mysyuk, Leyden Academy on Vitality and Ageing, Poortgebouw LUMC, Rijnsburgerweg 10, 2333 AA Leiden, the Netherlands. Phone: +3171 5240960. Email: mysyuk@leydenacademy.nl. Received 10 Oct 2012; revision requested 18 Nov 2012; revised version received 27 Nov 2012; accepted 4 Dec 2012. First published online 7 January 2013. already established. For instance, new institutional economic approaches on informal social norms propose that institutional frameworks can be incorporated or embedded in organizations and governing bodies. In other words, social institutions reflect the social arrangements in which they are based and incorporate the standards and expectations that are present within that society. Considering this background, it is indispensable to broaden the focus of elder abuse research and understanding from individual characteristics and situational circumstances of the victim or perpetrator to a focus that includes the environment in which older people live, interact, and communicate with other people and in which they receive care, help, and support.

In this paper, we explore the framing of elder abuse as a social problem and a health problem. It will also address how the changing culture of healthcare changes the role of older person in it. The scale of elderly who experience abuse 
draws more attention to the position of the elderly in society in general and the acceptable and expected help-seeking behavior from older persons in current healthcare systems. Through discussions about elder abuse of older persons and professionals involved in elder abuse in the Netherlands, we investigate factors related to social arrangements and the healthcare system, its organization, structure, and principles. The influence of environmental factors on older people is, therefore, crucial if we wish to understand and explain the circumstances of elder abuse.

\section{Methods}

A descriptive analysis of Dutch society and healthcare system was performed to provide more information about the changes in the Dutch society and the healthcare system, and better understanding of the discourse on elder abuse. Different studies about the Dutch healthcare system, its development, its shifts, and integration of neoliberal principles were analyzed.

A qualitative study was conducted to analyze discourse on elder abuse in interest groups dealing with elder abuse. The methods of data collection were focus groups and in-depth semi-structured interviews. The study was conducted among older persons, men and women aged 65 years and older, professional groups, and experts. The elderly were contacted through elderly advisors and were asked to participate in the research. The experts were approached through different organizations and contact persons via snowball sampling technique and a round mail for participation in a network for people who work and are involved in the field of elderly care. Confidentiality and anonymity were guaranteed through signing an informed consent form or having an explicit oral agreement.

\section{Focus groups}

The data for this paper is primarily based on eight focus groups. The topics that were discussed were defining elder abuse and the necessary actions that should be performed to prevent or intervene in the problem. We included different professional groups; experts, policy makers, managers, and older people themselves. In total, 42 participants were included. The focus groups lasted 1-2.5 hours, depending on the number of participants. The list with the potential participants for the focus groups was made on the basis of known organizations in the Dutch field of elder abuse. Then, persons from different organizations who are involved in the field of elderly care were included to the list using a snowball sampling technique (asking for further referral and potential participants). Following this, all the potential participants were contacted via emails and phone calls and invited to take part in a particular focus group based on their expertise, experience, and skills. The focus groups were not representative of the population in general as the group selection was not random and the sample was quite small but instead was intended to gather persons from diverse interest groups and diverse backgrounds as this study targeted to collect diversity of views and opinions.

All participants were informed of the purpose of the focus groups. Before the start of the focus groups, permission was asked for recording. The focus groups took place between February and March 2012. All groups were transcribed verbatim. Analysis was done primarily inductive with NVivo qualitative software according to the grounded theory approach as outlined by Glaser and Strauss (1967).

\section{Interviews}

In-depth qualitative interviews with older people about their opinions, attitudes toward elder abuse, and expert interviews with diverse professionals who are working in the field of elder abuse were conducted. The latter showed how the cases of abuse were identified, assisted, and followed-up.

For the semi-structured interviews two interview guides were used. The first one was interview guide for people who experienced elder abuse. The main topics of the interview guide for older people who experienced abuse were: demographic and social background, health status and daily life, care, experience with elder abuse, social network/help and support, and social life. The interviews lasted between 2 and 3 hours. Interviews for this group of older persons are still ongoing but the interviews discussed in this paper took place between April and August 2012.

The second interview guide was a topic list for experts professionals who work with older people, or who have experience in the field of elder abuse. The topics of the interview guide for experts included: background and meaning of elder abuse, profiles of victim and perpetrator, collaboration/network, perspectives of wider society, and necessary actions for dealing with the problem (see Appendix). The expert interviews lasted $1-1.5$ hour.

\section{Analysis}

Interviews were transcribed verbatim for analysis. Interview transcripts were stored securely on a USB-stick with the researcher and did not include personal identifiers. Verbatim transcripts of the interviews were then comprehensively 
and systematically analyzed using the computer software NVivo, a tool for analysis of qualitative data. The approach used for analyzing data was primarily inductive, where analytical concepts and perspectives are derived from the data through coding technique based on a grounded theory approach (Glaser and Strauss, 1967). Such an approach allows exploring the ways in which respondents explain their own experiences and also allows unexpected topics, issues, and thoughts to emerge.

\section{Results}

\section{Dutch society}

Pillarization based on religious or social-political affiliation, strong social control, and concurring traditions were prominent characteristics of Dutch society in the last centuries. The feeling of common responsibility, dependence on the government and social system, a relative lack of individual choices and personal autonomy were leading and important principles of politics and society. With time values that were important earlier changed or were modified and new ones are becoming more and more salient.

Contemporary Dutch society is based on the principles of a mixed neoliberal ideology that incorporates notion of individualism and personal freedom, independence, choice, responsibility, and competition. These features are also central in the neoliberal approach to healthcare; it emphasizes principles of self-sufficiency, responsibility, and independence in healthcare and encourages people to be responsible for their own lives and accountable for their actions and well-being. Hence, in a neoliberal approach responsibility for disease and health is placed on the individual. Additionally, the neoliberal individual is autonomous, selfreliant and self-concerned, and "free" of obligations to provide for the needs of others. Contrary to these neoliberal notions, healthcare is bound to interdependency and vulnerability. Furthermore, the patient's abilities of self-management and autonomy may be weakened, and the patient may feel both intimidated and powerless as he or she is unable to fulfill basic needs or functions (Ruthjersen, 2007).

\section{The Dutch healthcare system}

To understand the discourse on elder abuse presented below, we provide some background information about the Dutch healthcare system. The Dutch healthcare system is a hybrid system of public, private, and professional elements (Schrijvers, 1998; Boot and Knapen 2001; Putters 2001). According to the Dutch Constitution, the government is responsible for the accessibility, the quality, and the efficiency of healthcare. In spite of this, the government plays only a minor role in the implementation of these main principles of healthcare and, therefore, depends on the collaboration and cooperation of insurance companies, private institutions of healthcare, and other professional organizations which are dependent on each other. Already since the 1980s, when more market elements were introduced in the healthcare system, these developments have been widely debated. For instance, hospitals introduced different methods commonly used in businesses such as "social entrepreneurship," independence of the customer and centralization of the position of the manager (Picone et al., 1998; Grit and Dolfsma, 2002).

Current discourse in healthcare focuses on changes in the position of the patient, quality of care delivered, and leadership in healthcare organizations. Principles such as solidarity and equal access have long been guiding the Dutch healthcare system (ter Meulen and van der Made, 2000). However, instead of the collective responsibility that is characteristic of solidarity, an increased emphasis is now placed on the requirement for individuals to take care of their own healthcare needs. This emphasis on individual responsibility and personal autonomy can empower individuals to take care of their own needs, to arrange the necessary care services according to individual preferences and to take accountability for their own choices. However, individual responsibility finds its limits with vulnerable groups (elderly, children, individuals with psychological or mental disorders), for whom it is very difficult to realize personal responsibility for health, including financial responsibility. That is why the principle of individual responsibility in healthcare is important, but it has to be in balance with the principle of solidarity with vulnerable groups (ter Meulen and Maarse, 2008).

\section{Environmental factors in framing abuse and neglect}

We studied the transcripts to analyze how different professional groups and older persons themselves frame abuse. This included the circumstances under which abuse and neglect occurred, the explanation that was provided for why abuse occurred and continued, and the possible interventions. We discuss two main explanatory frameworks that 
were provided to frame abuse and neglect by our participants: social arrangements and healthcare systems. Both, as is clear, were pointing toward socio-structural factors that influence abuse and neglect. In our analysis, considering the social and healthcare changes as outlined above, we pay particular attention to how the framing of professionals and older persons reproduces, reflects, integrates, or contradicts political agendas that incorporate neoliberal principles.

\section{Social arrangements}

Despite that prevalence rates show no distinct increase or decrease in elder abuse incidence since it was brought under attention in the 1970s, participants in this research did voice concern that current changes in society, especially in regard to the position of older persons and social cohesion have led to an increase in abuse and neglect. In explaining elder abuse as influenced by social arrangements, diverse themes were touched upon: social taboo, social control and responsibility, and institutional cultures.

\section{A social taboo}

Both professionals and older persons discussed the limited knowledge and awareness of elder abuse and neglect among the general public. Explaining this lacuna was done by reference to the social taboo that surrounds discussing elder abuse and neglect. For older persons, elder abuse was something one rather not discussed: "it is not something you would like to talk about." This apprehension was seen as logical because of the feelings of shame and incompetence that were seen as related to its occurrence. The silence that surrounded it was according to older participants one of the reasons why it was not recognized easily. Professionals, especially in the group of managers and policy makers, voiced a similar idea. One manager stated it as follows: "Among elderly it [elder abuse] is still a taboo; a hidden problem and elderly have difficulty talking about it."

The question why this is still a taboo, and for some increasingly taboo, was explained by two social factors: the position of elderly and social expectations of self-reliance. One more individual factor was mentioned as explanatory factor for this taboo: the position of an older person within the individual life course.

The taboo sphere was first explained by the social position of older persons both in the past and nowadays. Generational differences in attitudes were mentioned by older persons themselves to explain why abuse was still taboo: "It is different generation, older people accept everything, they are glad about the things they receive, you don't create conflicts." Others phrased this attitude in a principle of "older persons do not wish to bother others." Both statements refer to a marginalized position of older persons within society at large.

This attitude of not wishing to be burdensome to others was not only framed as a generational difference, but also seen as something that run counter to social expectations. The occurrence of abuse and neglect was seen as a form of escalating dependence. Older persons stated that by saying one is abused one immediately became a vulnerable and dependent person. This was contrasted to the widespread imagery of older persons that are supposed to be embracing and choosing for "active ageing," a good old life and to be "successfully aged." This good old life is particularly portrayed in advertisements for pension schemes in the Netherlands, which are not unique for the Netherlands. In these advertisements, a rather young (mostly famous) retired person is portrayed in an attractive retirement setting: in a nice, fast car, on a remote island, or in a second house in a rural setting in France or Italy. The retiree is often arguing in favor of the pension scheme and phrases the "good old life" as something attainable for everyone and a choice. In the Netherlands, this kind of portrayal is identified as Het Zwitserleven gevoel (The Swiss life feeling) after one of the largest pension insurers that is particularly successful with this kind of commercials.

The taboo of elder abuse was further explained by contrasting it within the individual life course. Being abused meant for older participants that a situation of dependence and vulnerability was reached that was challenging a self-image over the life course of an independent adult. Thus, both from a social perspective and an individual perspective, expectations toward self-reliance and independence were undermined by the occurrence of abuse.

It is striking that in a life phase were dependence seems a natural and inescapable part, simply because physical decline becomes inevitable, the framing of this dependence is an explanatory factor in the exact taboo around abuse and neglect. It seems to refer to the social acceptability of dependence. A comparison with child abuse and neglect makes the tension inherent in this most apparent. Child abuse has been thoroughly on the agendas for some time now, and despite that there are some major and important differences in approach, it is quite illuminating to compare the situation of older persons that are mistreated with those of children. Children, as they are minors, are by extension seen as dependent and vulnerable, but more importantly expected to be dependent. In contrast, and this might seem straightforward, older 
adults are expected to be independent, because they are of age and have already led an independent life. The reversal from independence to dependence is filled with tension because from an individual perspective dependence is a break with the selfimage that has been built throughout the life course, and from a social perspective an adult should be self-reliant, especially in current political discourse about older persons.

In several measures to reduce healthcare costs of an increasing older population - and the Netherlands is probably not an exceptional case in this respect - measures such as saving for healthcare costs at older age, compulsory informal care, and even selling one's house to pay for healthcare are all referring to an individual responsibility to "take care of one's own old age." Especially recurrent in the discourse in the Netherlands about older persons and care is the concept of eigen regie (own control) in which healthcare is chosen, initiated, and organized by the healthcare recipient. Herewith the expectation toward older persons is changed from a passive receiver to an active coordinator. Even though this is, certainly for some older persons, a welcome change, it does make it hard to admit that one is no longer independent, competent, and responsible and admitting the opposite comes with "shame" and "humiliation" as older persons stated. In this explanation, the influence of a political discourse inspired by neoliberalism becomes clear: as older persons confronted with abuse they fail to fulfill and satisfy neoliberal principles of an individual who is autonomous, self-reliant, and selfresponsible.

\section{Social control and responsibility}

Besides a perceived social change to focus more on individual self-reliance and responsibility, and concurrent changes in the social position of older persons, a related but somewhat differently framed societal change was mentioned by our participants in explaining elder abuse: a decrease of social solidarity and responsibility. In the focus group of interest organizations of older persons, one participant phrased it as follows:

....It is how the entire elderly care works nowadays. Elderly are more and more left alone, in the individual situation. So there is little social control and I ask myself: how far should that go? Elderly themselves sometimes need and wish for more social control....

The decrease of social control and responsibility was considered important in preventing and in discovering elder abuse. Risk factors such as loneliness, disturbed family relations, and derailed care were considered to thrive well in a situation were social control and responsibility decreased.

There is no social control, no feedback. .. the social context is very important, because then you have social control, you keep an eye out for each other. That actually counts for every vulnerable person, children, older persons.... (GP in focus group)

Social control then functioned as a safety net in case situations escalated, in particular for lonely older persons and (over)burdened informal caregivers. Similarly, it was argued that social control and responsibility could step-in in situations when increasing physical dependence escalated in already fragile family relations. Elder abuse within families was considered to occur within a family history in which relationships were already shaped and influenced by (disturbed) power relations. As one older participant phrased it, "when family relationships are solidified in a certain pattern of behavior." Revenge from adult children for abuse occurring in their childhood was an especially vivid example of when accordingly "social control and responsibility" could have intervened. The described social change refers to a perceived individualism and the principle of being free of obligations to provide for others that is also put forward in neoliberal discourse, as outlined above.

\section{Institutional cultures}

A theme that recurred in several groups and interviews was a specific institutional culture. In some institutional settings such as residential care facilities and nursing homes this was viewed as a "culture" that allowed certain behavior that can be seen as abusive. A lack of transparency, for instance, to discuss burdened care professionals or colleagues that transgressed boundaries was sometimes mentioned. Some even argued that the acceptability of certain behavior was extended across what was termed as "acceptable boundaries" as professionals brought their own norms and values into these institutions and certain abusive behavior "[is] connected to your own norms and values, what you think is acceptable and what is not" (focus group GPs). What abuse is, and what not, where the boundary is, is difficult to assess. Professionals who discussed this issue in their focus groups did not reach a consensus about this. Some felt it was when an older person "experienced harm" or felt "boundaries were crossed" and whether that might be feeling offended or experiencing harsh treatment was irrelevant. Others felt that the label abuse required more, but what exactly demarcated the boundary of abuse and where it lied was left open. This "gray zone" of abuse is something that 
cannot be resolved easily; perhaps a doubt about the acceptability of certain behavior by any of the involved parties should already be enough for discussion.

Some even argued that the current healthcare system was actually a form of abuse, stating that "abuse is inherent in the culture of institutions" (focus group elderly) and "is a bit in the culture [...] and think that this kind of treatment is fine" (focus group institutional care). The idea that current healthcare is conducive for an environment of abuse, or even is a form of abuse by itself, was remarked by some of the participants. As one of the interviewees phrased it:

What is not abuse? A lot of things are not seen as abuse, which are actually abuse. They are only put in such a cultural context that they are seen and perceived differently. The whole health care system has to be changed. You have a lot of abuse in health care. Abuse in the health care already begins in the emergency department when doctors are not willing to deal with elderly [...]. The needs of older people are not a priority, money is the priority. (Focus group elderly)

This respondent stated it rather strongly, but some of the other older interviewees also felt that older persons were neglected within the system and were perceived as difficult and burdensome and that this by itself was a form of abuse. Others felt that perhaps care professionals were not really to blame, but that the current system in which work pressure is high and individual attention is under pressure was enabling a form of neglect and an environment in which abuse occurred as a result of stress and a lack attention for the social dimension of care.

\section{Healthcare system}

The liability of the healthcare system itself in relation to institutional cultures that was mentioned by some of our respondents leads us to the way the role of the healthcare system itself was framed by our participants. As outlined above, in the explanatory framework referring to social arrangements elder abuse was seen as a social problem that is to be dealt with in broader society. In the explanatory framework discussed in the following paragraphs, however, elder abuse was framed as a health problem that involved the healthcare system.

The major reforms such as outlined earlier in this paper have led to some large changes in the provision, financing, planning, and monitoring of care for older persons and especially vulnerable persons. Although it is unclear up to what level older person participate in the healthcare system, it can be expected that at least for some of the older persons involved healthcare systems are an important entry point for seeking help and assistance. In the Netherlands, on average older persons visit the GP eight times a year and vulnerable elderly visit the GP more often than this average (van Campen, 2011). Notwithstanding that there are numerous cases in which healthcare is actually largely avoided and only involved when the situation escalates. Thus, for as far as older people who are abused participate in the healthcare system, participants felt that changes in the system were creating an environment in which abuse can occur and falls "through the cracks." Two broad aspects, which are mutually interdependent, of the changes in the system were brought to the fore: fragmentation of care and changes in the financing of healthcare.

\section{Fragmentation}

Healthcare systems can be viewed in diverse ways. Whether seen as reaching from primary to tertiary care or from informal to formal care, numerous providers and institutions are involved in caring for the same persons. According to prevalence rates vulnerable elderly with for instance decreased mobility, loneliness, or cognitive decline are at highest risk for elder abuse. They are also the ones that visit several healthcare institutions at the same time leading to a rather diffuse picture of who is overall responsible or has to take the coordinating role in providing help for older persons experiencing abuse. Professionals involved in caring for older persons spoke about a lack of cooperation or coordination among diverse healthcare organizations and levels that sometimes perpetuated a situation of abuse. As one participant in the focus group of outpatient care discussed:

Once we had an older woman, she was placed inside a residential care facility after the situation at home had become unbearable. When I arrived at the residential care facility I discovered that the caretaker who was responsible for the abuse was actually being involved in the care plan of the victim. There had not been an exact exchange of information so the situation could continue inside the home.

Whether this case is representative remains unclear as specific quantitative data is lacking. However, the mechanisms exposed, a fragmentation of care which results in a loss of information was confirmed by other participants as well. Thus, one GP discussed a similar situation and said "the whole transfer of the client from a problematic home situation to a residential care home is not happening in a proper way.... Problems in the system are appearing again." The system referred to in this case was mainly pointing toward the organization of care in which diverse institutions have to cooperate in one 
case. The multiplex and multidisciplinary problems that come with a case of elder abuse, that can range from physical problems, family problems, personality and psychological problems, and so on leads to an involvement of several organizations. The solution is no less fragmented as it requires additional involvement of care, but sometimes also a social worker, outpatient care and in some cases psychological care. The experience of the system as something external to the individual care provider is related to the feeling that influencing this system often goes beyond the individual care professional or organizations:

You need diverse partners to get things done, because once outside of the health care system I will no longer have an eye on the client. On the older woman and how she is doing. So well, we encounter heavy, rather complex cases. We actually encounter poignantly enough that increasingly more often, and that is sad.... (Focus group outpatient care)

Some of the participants tried to combat these problems in setting up network meetings in which diverse medical, paramedical, and social service representatives participate. In framing elder abuse a concern for healthcare, and by extension also as a health problem, it also justified intervention and supervision under this label. The implications of framing it under this label go beyond this article. It is important, however, to mention that by treating it as a health concern, a prime, universal concern, certain actions become justifiable, acceptable, and legitimate under the label of the integrity of health (in contrast to framing it as a social problem). Others sought the solution for a lack of coordination on the local level on a higher level, one expert interviewee stated that "the government has to determine the structure of elderly care; it should be responsible for monitoring the care system and give clear protocols. This can make changes."

\section{Financing}

Perhaps a more specific factor for the Netherlands that is interrelated with the fragmentation of care is the financing of care. Since 2010, care organizations are preparing and slowly anticipating a change in which financing of care under some specific laws is shifted from the national governmental level to the municipal level. Important in the debate about elder abuse is the shift in responsibility for the allocation of care that is given under the social support act to the municipality. This act provides among other things provision of facilities, help, or support for persons that are disabled in some way. The law includes support for older persons and covers, for instance, home care, but also a wheel chair or transport. The general idea is that this will enable a better assessment of the needs and demands on community scale. Participants, however, perceived this change to lead to a further fragmentation of care for elder abuse, exactly because financing means and distribution remained unclear:

Now it is dependent on the municipalities, on the municipality in which you live and what is currently the priority within this municipality. How poor is this municipality? How social is this municipality? And which choices does this municipality make? (Focus group outpatient care)

The uncertainty about whether certain interventions would be financed and diverse priorities of individual municipalities was also a concern for participating elderly. As one of the participants in that focus group for elderly stated rather boldly: "For municipalities, it is now a choice between street lightening or elder abuse intervention." Even if it is somewhat doubtful that this was the exact case, as care and infrastructure facilities do come from a different budget, however, it was perceived and felt this way by the older participant. This statement, however, seemed to refer much more to a questioning of the capabilities and competence of municipalities to decide on the allocation of money within the framework of the social support act. Some case managers discussed the difficulty they had in working within the given budget. In practice, a certain amount of hours is allocated for each case of abuse, but sometimes there are more cases of abuse or there are certain particularly difficult cases that require more investment. Money, however, had run out in the meantime. Some care professionals, therefore, resorted to shopping around, to put it rather simply, and asked for the involvement of long-term care facilities to be able to apply for budget as part of the Act for Long-Term Care, which again led to a further fragmentation as the case had to be handed over.

Decentralization in both care organization and financing, also partly inspired by neoliberal tenets, might seem a way of increasing a fit between needs and provision, but for a multiplex and multidisciplinary problem, there is no specific financing structure. By framing it as a health problem, the involvement of a wide variety of organization and care acts was legitimized. This also enabled, conversely, a further fragmentation of practices, which participants felt were sometimes responsible for prolonging situations of abuse.

\section{Conclusion}

In this paper, we have discussed the way healthcare professionals and older persons frame elder abuse. 
The environmental factors appeared important in framing of abuse. We have shown that two explanatory frameworks were most prominent in the discourse studied; on the one hand social arrangements and on the other hand factors in the healthcare system. In the first, elder abuse was foremost framed as a social problem. In the last, elder abuse was primarily framed as a health problem. Especially the social problem referred to neoliberal principles of an autonomous, selfreliant, and self-responsible individual. Prominent in the framework of healthcare was more the conceptualization of a system that went beyond the individual healthcare provider. Current changes in healthcare system that were mentioned as impacting practices and intervention in the area of elder abuse were decentralization in organization and financing.

For healthcare professionals and providers, it is important to be aware how a phenomenon such as elder abuse is framed as it can have important implications (as became apparent in the discourse of older persons) for help seeking behavior and the way the problem is expressed. Therefore, it is equally important to be aware of how different professional groups frame elder abuse as it affects the way they navigate through the healthcare system in seeking help for their affected older person. Being aware of differences in conceptualization can help to create a better understanding between healthcare professionals and older persons.

\section{Conflict of interest}

None.

\section{Description of authors' roles}

All three authors contributed equally to the thoughts expressed in this paper. Yuliya Mysyuk and Jolanda Lindenberg wrote the draft of the paper, whereas Rudi Westendorp assisted and contributed to the final manuscript.

\section{References}

Boot, J. M. and Knapen, M. H. J. M. (2001). Handboek Nederlandse Gezondheidszorg (Handbook Dutch Health Care). Utrecht: Het Spectrum.

Glaser, B. G. and Strauss, A. (1967). The Discovery of Grounded Theory: Strategies for Qualitative Research. Chicago, IL: Aldine transaction.

Grit, K. and Dolfsma, W. (2002). The dynamics of the Dutch health care system - a discourse analysis. Review of Social Economy, 3, 377-401.

Hörl, J. (2010). The perpetrator-victim dichotomy in elder abuse reconsidered. Elder abuse symposium. Leiden, 2010.

Lachs, M. S. and Pillemer, K. (2004). Elder abuse. Lancet, 346, 1263-1272.

Picone, G., Uribe, M. and Wilson, R. (1998). The effect of uncertainty on the demand for medical care, health capital and wealth, Fournal of Health Economics, 17, 171-185.

Ruthjersen, A. (2007). Neo-Liberalism and Health Care. Brisbane, Australia: Queensland University of Technology.

Schrijvers, A. J. P. (ed.) (1998). Health and Health Care in the Netherlands. Maarssen: Elsevier/De Tijdstroom.

ter Meulen, R. and Maarse, H. (2008). Increasing individual responsibility in Dutch health care: is solidarity losing ground? Fournal of Medicine and Philosophy, 33, 262-279.

ter Meulen, R. and van der Made, J. (2000). The extent and limits of solidarity in Dutch health care. International fournal of Social Welfare, 9, 250-60.

van Campen, C. (ed.) (2011). Kwetsbare Ouderen (Frail Elderly). Den Haag: SCP. 